\title{
Spokes cluster: The search for the quiescent gas ${ }^{\star}$
}

\author{
J. E. Pineda ${ }^{1,2}$ and P. S. Teixeira ${ }^{3,4}$ \\ ${ }^{1}$ European Southern Observatory (ESO), 85748 Garching, Germany \\ 2 UK ARC Node, Jodrell Bank Centre for Astrophysics, School of Physics and Astronomy, University of Manchester, Manchester, \\ M13 9PL, UK \\ e-mail: jaime.pineda@manchester.ac.uk \\ 3 Universität Wien, Institut für Astrophysik, Türkenschanzstrasse 17, 1180 Vienna, Austria \\ e-mail: paula.teixeira@univie.ac.at \\ ${ }^{4}$ Laboratório Associado Instituto D. Luiz-SIM, Universidade de Lisboa, Campo Grande, 1749-016 Lisboa, Portugal
}

Received 6 December 2012 / Accepted 25 April 2013

ABSTRACT

\begin{abstract}
Context. Understanding the role of turbulent and thermal fragmentation is one of the most important current questions of star formation. To better understand the process of star and cluster formation, we need to study in detail the physical structure and properties of the parental molecular cloud. In particular, it is important to understand the fragmentation process itself; this may be regulated by thermal pressure, magnetic fields, and/or turbulence. The targeted region, the Spokes cluster, or NGC 2264-D, is a rich protostellar cluster where previous $\mathrm{N}_{2} \mathrm{H}^{+}(1-0)$ observations of its dense cores presented linewidths consistent with supersonic turbulence. However, the fragmentation of the most massive of these cores appears to have a scale length consistent with that of the thermal Jeans length, suggesting that turbulence was not dominant.

Aims. These two results (derived from $\mathrm{N}_{2} \mathrm{H}^{+}(1-0)$ observations and measurements of the spatial separations of the protostars) probe different density regimes. Our aim is to determine if there is subsonic or less-turbulent gas (than previously reported) in the Spokes cluster when probing higher densities, which would reconcile both previous observational results. To study denser gas it is necessary to carry out observations using transitions with a higher critical density to directly measure its kinematics.

Methods. We present APEX $\mathrm{N}_{2} \mathrm{H}^{+}(3-2)$ and $\mathrm{N}_{2} \mathrm{D}^{+}$(3-2) observations of the NGC 2264-D region to measure the linewidths and the deuteration fraction of the higher density gas. The critical densities of the selected transitions are more than an order of magnitude higher than that of $\mathrm{N}_{2} \mathrm{H}^{+}(1-0)$.

Results. We find that the $\mathrm{N}_{2} \mathrm{H}^{+}(3-2)$ and $\mathrm{N}_{2} \mathrm{D}^{+}$(3-2) emission present significantly narrower linewidths than the emission from $\mathrm{N}_{2} \mathrm{H}^{+}(1-0)$ for most cores. In two of the spectra, the nonthermal component is close (within 1- $\sigma$ ) to the sound speed. In addition, we find that the three spatially segregated cores for which no protostar had been confirmed show the highest levels of deuteration.

Conclusions. These results show that the higher density gas, probed with $\mathrm{N}_{2} \mathrm{H}^{+}$and $\mathrm{N}_{2} \mathrm{D}^{+}(3-2)$, reveals more quiescent gas in the Spokes cluster than previously reported. More high-angular resolution interferometric observations using high-density tracers are needed to truly assess the kinematics and substructure within NGC 2264-D.
\end{abstract}

Key words. ISM: clouds - stars: formation - ISM: molecules - ISM: individual objects: NGC 2264-D

\section{Introduction}

The current paradigm of cluster formation starts with a highly turbulent cloud in which dense clumps ${ }^{1}$ are close to virial equilibrium (e.g., McKee \& Ostriker 2007; Bate 2009). This turbulence will decay in a free-fall time if there is no driving mechanism. Theoretical studies show that protostellar outflows might be one mechanism capable to drive turbulence in the cloud (e.g. Li \& Nakamura 2006), which agrees with some observational results (Palau et al. 2007; Fontani et al. 2012). However, other studies have shown that outflows might not have enough energy and momentum to sustain turbulence (Arce et al. 2010), while shells driven by young stars might (Arce et al. 2011).

$\star$ This publication is based on data acquired with the Atacama Pathfinder Experiment (APEX) under programme 088.F-9322A. APEX is a collaboration between the Max-Planck-Institut für Radioastronomie, the European Southern Observatory, and the Onsala Space Observatory.

1 Regions that may contain substructure and where several stars might form.
NGC 2264, located $\sim 800$ pc away (between 750-913 pc, Dahm \& Simon 2005; Baxter et al. 2009; Naylor \& Mayne 2010), is a well-studied cluster in which we can study the role of turbulence in star formation. Within this cluster there is a rich grouping of protostars, known as NGC 2264D or as the Spokes cluster (we use both nomenclatures interchangeably in this paper). This region has been observed at (sub)millimeter wavelengths with JCMT/SCUBA and IRAM/30m, where several dense cores were identified (Ward-Thompson et al. 2000; Williams \& Garland 2002; Wolf-Chase et al. 2003; Peretto et al. 2006). Spitzer observations of the region revealed an agglomeration of deeply embedded protostars (Class 0/I sources) identified primarily at $24 \mu \mathrm{m}$. Teixeira et al. (2006), using a nearestneighbor analysis, found that these protostars have a characteristic projected spacing, $20 \pm 5^{\prime \prime}$, which is consistent with the thermal Jeans length of the region, $27^{\prime \prime}$ or $0.104 \mathrm{pc}$ at the distance of $800 \mathrm{pc}$ (calculated using the mean filament density of $3 \times 10^{4} \mathrm{~cm}^{-3}$ from Williams \& Garland 2002; and temperature of $17 \mathrm{~K}$ from Ward-Thompson et al. 2000). Since the protostars are embedded in dense material, and the virial mass of the system 
is lower than the total gas and dust mass (Williams \& Garland 2002; Wolf-Chase et al. 2003; Peretto et al. 2006), the protostars are bound to the filaments and are very likely still tracing the primordial substructure of the cluster. The measured regular spacing (consistent with the thermal Jeans length) of the protostars is thus interpreted as a fossil signature of thermal fragmentation of the parental filament (Teixeira et al. 2006).

Peretto et al. (2006) also observed the 15 cores in the Spokes cluster using dust continuum emission. These dense cores present a mean $\mathrm{H}_{2}$ density ranging between $10^{5}$ and $10^{6} \mathrm{~cm}^{-3}$, and masses of 1.8 to $17.3 M_{\odot}$ (Wolf-Chase et al. 2003; Peretto et al. 2006). Direct comparison with the Spitzer $24 \mu \mathrm{m}$ image shows that the cores identified by Peretto et al. (2006) have $24 \mu \mathrm{m}$ Spitzer counterparts (i.e., they harbor protostars) except for DMM-8 and DMM-11. Peretto et al. (2006) also observed these 15 dense cores in $\mathrm{N}_{2} \mathrm{H}^{+}(1-0)$, which traces highdensity gas $\left(2 \times 10^{5} \mathrm{~cm}^{-3}\right)$, and found only lines with supersonic velocity dispersions (Mach $\sim 3$ ), which is at odds with the interpretation of thermal Jeans fragmentation. Follow-up observations by Teixeira et al. (2007) using the Submillimeter Array (Ho et al. 2004) of one of the cores, DMM-1, showed that it had fragmented into seven compact $1.3 \mathrm{~mm}$ sources, of masses ranging between $0.4 M_{\odot}$ and $1.2 M_{\odot}$. The mean separation of these sources within DMM-1 is consistent with the thermal Jeans length of the core, which indicates that the core underwent thermal fragmentation.

The apparent disagreement between the observation of supersonic turbulence in the dense gas and the thermal fragmentation, suggested by the spatial separation of the young stellar objects (YSOs), can be tested. If thermal fragmentation indeed took place in the star formation process in this cluster, low levels of turbulence should still be seen using a higherdensity tracer. The Spokes cluster contains high-density gas over an extended region, which could explain the $\mathrm{N}_{2} \mathrm{H}^{+}$(1-0) observations by Peretto et al. (2006), which show supersonic turbulence in a dense gas tracer. These observations might not be tracing the densest gas in the star-forming cores, but instead it traces the dense environment containing the entire region. This interpretation can be readily tested with new observations using an even higher-density tracer at similar angular resolutions: $\mathrm{N}_{2} \mathrm{H}^{+}(3-2)$ or $\mathrm{N}_{2} \mathrm{D}^{+}(3-2)$. Moreover, the $\left[\mathrm{N}_{2} \mathrm{D}^{+} / \mathrm{N}_{2} \mathrm{H}^{+}\right]$abundance ratio is enhanced in the densest and coldest regions of cores (Caselli 2002), and it decreased after the protostar was formed (Emprechtinger et al. 2009). Therefore, the $\mathrm{N}_{2} \mathrm{D}^{+}(3-2)$ observations trace more pristine conditions than $\mathrm{N}_{2} \mathrm{H}^{+}(3-2)$.

A decrease in the velocity dispersion is expected when going to higher-density tracers, as shown by Myers (1983), Goodman et al. (1998), and Fuller \& Myers (1992) in regions with lower average densities. Moreover, Pineda et al. (2010) recently presented observations of dense gas as traced by $\mathrm{NH}_{3}(1,1)$ where a sharp transition from supersonic to subsonic turbulence is seen in the velocity dispersion. This result suggests that to form lowmass stars, the turbulence needs to be dissipated before the star can be formed. It is possible that in more massive and denser environments this transition would occur at higher densities, and therefore higher-density tracers need to be used. Foster et al. (2009) showed, from their observations of the dense cores in the Perseus molecular cloud, that the cores have subsonic $\mathrm{NH}_{3}(1,1)$ linewidths regardless of their stellar content or cluster environment. Moreover, even though the effect of the molecular outflows is important in the dynamics of the low-density gas, the dense gas is only locally affected (Pineda et al. 2010, 2011, and in prep.).

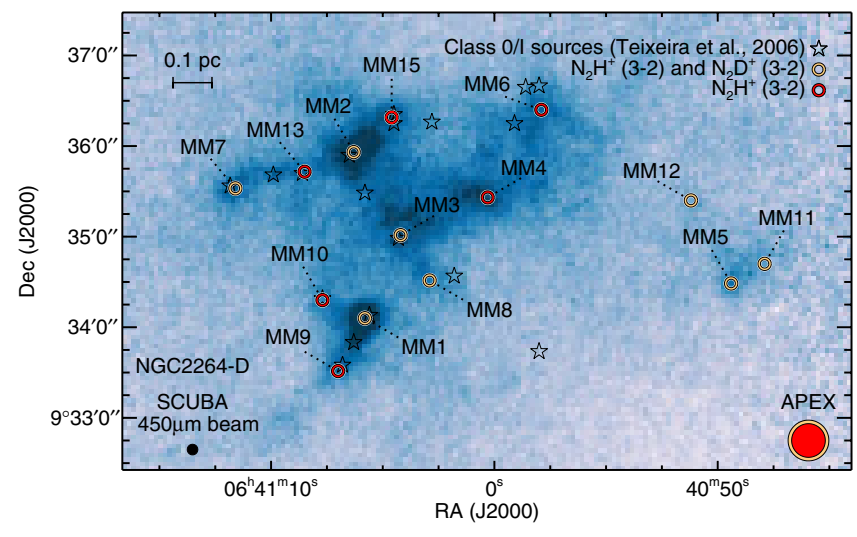

Fig. 1. SCUBA $450 \mu \mathrm{m}$ dust-continuum map for the Spokes cluster (Wolf-Chase et al. 2003). The observed dense cores identified in Peretto et al. (2006) are shown by the circles at their centers (color-coded according to which molecular line tracers were used in their observation). For comparison, the APEX beams corresponding to our observations are also shown in the lower right corner (following the same color-code convention).

Therefore, by observing the Spokes cluster in a denser-gas tracer than $\mathrm{N}_{2} \mathrm{H}^{+}(1-0)$, we can determine whether the emission arising from denser regions is less turbulent. This could provide a test of the thermal-fragmentation hypothesis of the quiescent gas.

\section{Data}

The observations of $\mathrm{N}_{2} \mathrm{H}^{+}$and $\mathrm{N}_{2} \mathrm{D}^{+}(3-2)$ molecular lines were carried out on 5 September and 4, 8-9, and 11-13 November 2011 with APEX. Every dense core in the Spokes cluster identified by Peretto et al. (2006) that presented a good detection of the $\mathrm{N}_{2} \mathrm{H}^{+}(1-0)$ line (14 cores) was observed in $\mathrm{N}_{2} \mathrm{H}^{+}(3-2)$ using the APEX-2 receiver of the Swedish Heterodyne Facility Instrument (SHeFI; Vassilev et al. 2008). The cores with the narrowest $\mathrm{N}_{2} \mathrm{H}^{+}(1-0)$ lines (eight cores) were also observed in $\mathrm{N}_{2} \mathrm{D}^{+}(3-2)$ using the APEX-1 receiver of the SHeFI (Vassilev et al. 2008).

Figure 1 shows a JCMT/SCUBA $450 \mu$ m map of the Spokes cluster, including the locations of the previously identified protostars and our APEX observations. A summary of the sources is listed in Table 1. The observed transitions, their spectroscopic properties, and observational parameters are listed in Table 2. We use the following nomenclature for source identification: MM??, where "??" is the source number (e.g., source D-MM1 of Peretto et al. (2006) is referred to as MM01 hereafter).

The observations used position-switching, with the offposition at $\left(\alpha_{\mathrm{J} 2000}=06^{\mathrm{h}} 40^{\mathrm{m}} 52.57^{\mathrm{s}}, \delta_{\mathrm{J} 2000}=09^{\circ} 32^{\prime} 36.5^{\prime \prime}\right)$. They were calibrated by regularly measuring the sky brightness and cold/hot loads in the cabin. All observations used the eXtended bandwidth Fast Fourier Transform Spectrometer (XFFTS), which provides two $2.5 \mathrm{GHz}$ units, with 32768 channels each unit and $1 \mathrm{GHz}$ overlap. This configuration provides an spectral resolution of $0.082 \mathrm{~km} \mathrm{~s}^{-1}$ and $0.099 \mathrm{~km} \mathrm{~s}^{-1}$ for $\mathrm{N}_{2} \mathrm{H}^{+}$ and $\mathrm{N}_{2} \mathrm{D}^{+}$(3-2), respectively.

All spectra were reduced using CLASS90 ${ }^{2}$. Each spectrum had a first- or second-order polynomial fitted to remove the baseline before averaging all scans.

2 http://www.iram.fr/IRAMFR/GILDAS 
Table 1. List of observed sources.

\begin{tabular}{|c|c|c|c|c|c|c|}
\hline \multirow[b]{2}{*}{ Source } & \multirow[b]{2}{*}{$\begin{array}{c}\text { RA } \\
\text { (hh:mm:ss) }\end{array}$} & \multirow[b]{2}{*}{$\begin{array}{c}\text { Dec } \\
\text { (dd:mm:ss) }\end{array}$} & \multirow[b]{2}{*}{$\begin{array}{c}\sigma_{v}{ }^{a} \\
\left(\mathrm{~km} \mathrm{~s}^{-1}\right)\end{array}$} & \multicolumn{2}{|c|}{ On-source time } & \multirow[b]{2}{*}{$\begin{array}{c}\text { \# YSOs } \\
\text { within APEX beams } \\
\end{array}$} \\
\hline & & & & $\begin{array}{c}\mathrm{N}_{2} \mathrm{H}^{+}(3-2) \\
(\min )\end{array}$ & $\begin{array}{c}\mathrm{N}_{2} \mathrm{D}^{+}(3-2) \\
\quad(\min )\end{array}$ & \\
\hline MM01 & 06:41:05.8 & $+09: 34: 06$ & 0.59 & 4.0 & 23.0 & $1(7)^{c}$ \\
\hline MM02 & $06: 41: 06.3$ & $+09: 35: 56$ & 0.51 & 2.3 & 28.0 & 1 \\
\hline MM03 & $06: 41: 04.2$ & $+09: 35: 01$ & 0.26 & 3.3 & 24.0 & 1 \\
\hline MM04 & $06: 41: 00.3$ & $+09: 35: 26$ & 0.81 & 3.3 & $\ldots$ & $0(1)^{d}$ \\
\hline MM05 & $06: 40: 49.4$ & $+09: 34: 29$ & 0.42 & 13.4 & 24.0 & $0(1)^{d}$ \\
\hline MM06 & $06: 40: 57.9$ & $+09: 36: 24$ & 0.55 & 3.3 & $\ldots$ & $0(1)^{d}$ \\
\hline MM07 & $06: 41: 11.6$ & $+09: 35: 32$ & 0.59 & 3.3 & 17.0 & 1 \\
\hline MM08 & $06: 41: 02.9$ & $+09: 34: 31$ & 0.34 & 3.3 & 31.0 & 0 \\
\hline MM09 & $06: 41: 07.0$ & $+09: 33: 31$ & 0.55 & 3.3 & $\ldots$ & 1 \\
\hline MM10 & $06: 41: 07.7$ & $+09: 34: 18$ & 0.72 & 3.3 & $\ldots$ & 1 \\
\hline MM11 & $06: 40: 47.9$ & $+09: 34: 42$ & 0.30 & 13.7 & 33.0 & 0 \\
\hline MM12 & $06: 40: 51.2$ & $+09: 35: 24$ & 0.38 & 8.0 & 79.0 & $0(1)^{d}$ \\
\hline MM13 & $06: 41: 08.5$ & $+09: 35: 43$ & 0.89 & 6.3 & $\ldots$ & 1 \\
\hline MM15 & $06: 41: 04.6$ & $+09: 36: 19$ & 0.64 & 13.7 & $\ldots$ & 2 \\
\hline
\end{tabular}

Notes. ${ }^{(a)}$ Velocity dispersion from Peretto et al. (2006) derived using $\mathrm{N}_{2} \mathrm{H}^{+}(1-0)$ fits. ${ }^{(b)}$ Number of protostars located within the APEX HPBW (see Table 2) (Peretto et al. 2006, and references therein), (Teixeira et al. 2006). ${ }^{(c)}$ Targeted SMA observations of this core revealed additional compact millimeter sources undetected in the infrared (Teixeira et al. 2007). ${ }^{(d)}$ The value in parenthesis corresponds to the number of faint $24 \mu \mathrm{m}$ sources that have no other wavelength counterpart (and therefore we cannot confirm their protostellar nature).

Table 2. Observed transitions and observational parameters.

\begin{tabular}{lccccccc}
\hline \hline Transition & $\begin{array}{c}\text { Frequency } \\
(\mathrm{GHz})\end{array}$ & $\begin{array}{c}H P B W \\
\left({ }^{\prime \prime}\right)\end{array}$ & $\begin{array}{c}\text { channel spacing } \\
(\mathrm{kHz})\end{array}$ & $\begin{array}{l}\left.\mathrm{km} \mathrm{s}_{\mathrm{MB}}\right) \\
\mathrm{N}\end{array}$ & $\begin{array}{c}B \\
(\mathrm{MHz})\end{array}$ & $\begin{array}{c}\mu \\
(\mathrm{D})\end{array}$ \\
\hline $\mathrm{N}_{2} \mathrm{D}^{+}(3-2)$ & 231.3219119 & 27.0 & 76.29 & 0.0989 & 0.75 & 38554.719 & 3.4 \\
$\mathrm{~N}_{2} \mathrm{H}^{+}(3-2)$ & 279.511862 & 22.3 & 76.29 & 0.0818 & 0.74 & 46586.867 & 3.4 \\
\hline
\end{tabular}

Notes. $\eta_{\mathrm{MB}}$ is the main-beam efficiency of the APEX telescope, $B$ is the rotational constant of the molecule, and $\mu$ is the dipole moment of the molecule.

\section{Results}

We detected $\mathrm{N}_{2} \mathrm{H}^{+}(3-2)$ and $\mathrm{N}_{2} \mathrm{D}^{+}(3-2)$ emission toward all 14 and 8 observed cores respectively. The $\mathrm{N}_{2} \mathrm{H}^{+}(3-2)$ and $\mathrm{N}_{2} \mathrm{D}^{+}$(3-2) spectra are shown in Figs. 2 and 3, respectively. In addition, we overplot on these figures the best-fit profiles to the lines.

The $\mathrm{N}_{2} \mathrm{H}^{+}$and $\mathrm{N}_{2} \mathrm{D}^{+}(3-2)$ lines were fitted in IDL $^{3}$ using a forward-fitting model that takes into account all hyperfine components, with the minimization performed using the mpfitfun program (Markwardt 2009). For a given centroid velocity $\left(v_{\mathrm{LSR}}\right)$, velocity dispersion $\left(\sigma_{v}\right)$, and total optical depth $\left(\tau_{\text {main }}\right)$ we computed the optical depth at each velocity as

$\tau=\tau_{\text {main }} \Sigma_{i} w_{i} \exp -\left(v-v_{i}-v_{\mathrm{LSR}}\right)^{2} /\left(2 \sigma_{v}^{2}\right)$,

where $w_{i}$ and $v_{i}$ are the relative weight and offset velocity for each hyperfine component. The model spectrum is then generated as

$T_{\mathrm{MB}}=\eta_{\mathrm{f}}\left[J\left(T_{\mathrm{ex}}\right)-J\left(T_{\mathrm{bg}}\right)\right]\left[1-\mathrm{e}^{-\tau}\right]$

where $T_{\mathrm{MB}}$ is the main-beam temperature, $\eta_{\mathrm{f}}$ is the filling fraction of the emission in the beam, $T_{\mathrm{ex}}$ is the excitation temperature, $T_{\mathrm{bg}}$ is the background brightness temperature $(2.73 \mathrm{~K})$, and

$J(T)=\frac{h v}{k} \frac{1}{\exp (h v / k T)-1}$.

\footnotetext{
3 Interactive data language.
}

Since the extent of the emission is unknown and it is unclear if it is more or less extended than the continuum emission, we assumed a filling fraction of unity, $\eta_{\mathrm{f}}=1$. Therefore, $T_{\mathrm{ex}}$ is obtained from the fit when $\tau_{\text {main }}$ is well constrained. We also compared the results of fitting the spectra using the HFS mode within CLASS90, where we obtained the same results within the fitted parameter uncertainties. This procedure usually returns well-constrained parameters, but when the total optical depth is poorly constrained $\left(\tau<3 \sigma_{\tau}\right)$, we kept the excitation temperature as a fixed parameter. For the $\mathrm{N}_{2} \mathrm{H}^{+}(3-2)$ line, the excitation temperature was set to the average value obtained from all other spectra, i.e., $6.7 \mathrm{~K}$, because this average excitation temperature provides the typical excitation conditions in the region. For $\mathrm{N}_{2} \mathrm{D}^{+}(3-2)$, the fit is only poorly constrained and therefore the excitation temperature was set to the value obtained from the $\mathrm{N}_{2} \mathrm{H}^{+}(3-2)$ fit, because both transitions are optically thin and have similar critical densities. This procedure is similar to that followed in Crapsi et al. (2005).

For most of the cores the fits are good, but there are two cores (MM03 and MM13) that present two distinct components along the line-of-sight. In this case two independent lines were fitted simultaneously, and each of the two components was labeled "a" or "b". There are two other cores, MM08 and MM04, which show a clear asymmetry in the line profile that is suggestive of an additional component along their line-of-sight. Unfortunately, we were unable to separate the blended components. Table 3 shows the results of the hyperfine fitting procedure to the $\mathrm{N}_{2} \mathrm{H}^{+}(3-2)$ and $\mathrm{N}_{2} \mathrm{D}^{+}(3-2)$ lines of all sources. 

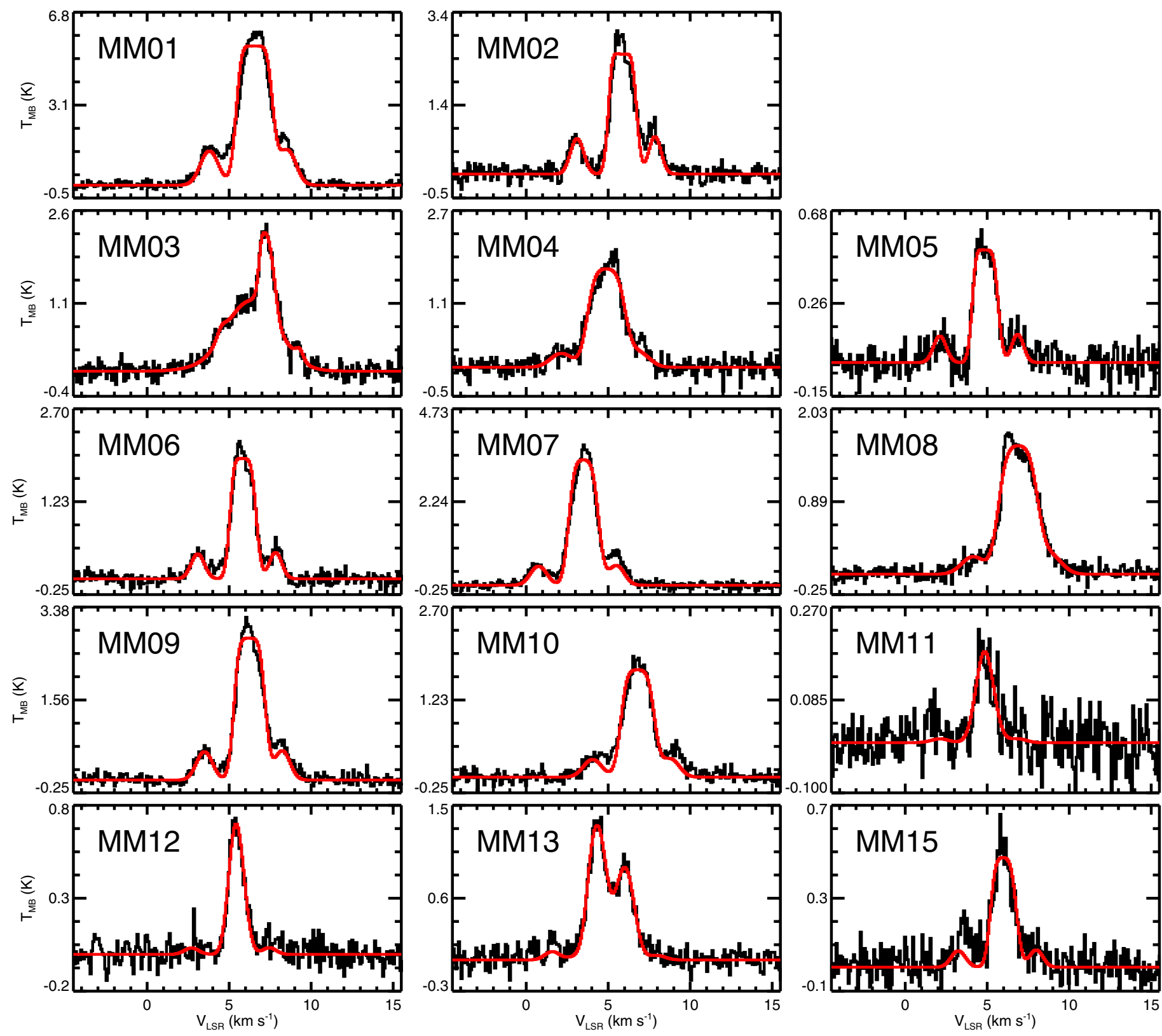

Fig. 2. Observed $\mathrm{N}_{2} \mathrm{H}^{+}(3-2)$ spectra toward the sources. The red curve is the best-fit model to the observations, whose fit parameters are shown in Table 3.

The column densities were calculated using the constant excitation temperature approximation and that the beam filling factor is 1 for both lines, see Eqs. (A.1) and (A.2) from Appendix A, which are based on Caselli et al. (2002).

Figure 4 compares the velocity dispersions obtained from the different molecular lines that we observed and the $\mathrm{N}_{2} \mathrm{H}^{+}(1-0)$ velocity dispersions reported in Peretto et al. (2006). The velocity dispersion, $\sigma_{v}$, has a thermal, $\sigma_{\mathrm{T}}$, and a nonthermal, $\sigma_{\mathrm{NT}}$, component and is given as $\sigma_{v}=\sqrt{\sigma_{\mathrm{T}}^{2}+\sigma_{\mathrm{NT}}^{2}}$. Each panel also indicates, for comparison purposes, expected velocity dispersions whose nonthermal components are $\sigma_{\mathrm{NT}}=c_{\mathrm{S}}(10 \mathrm{~K})$ (dashed lines) and $\sigma_{\mathrm{NT}}=2 c_{\mathrm{s}}(10 \mathrm{~K})$ (dotted lines). Figure 4a shows the comparison of the velocity dispersion obtained from the two different transitions $\mathrm{N}_{2} \mathrm{H}^{+}(1-0)$ and (3-2); the plot clearly shows that there is a trend for the velocity dispersion to be narrower for the higher-J transition. Figure $4 \mathrm{~b}$ shows the comparison of the velocity dispersions of the $\mathrm{N}_{2} \mathrm{H}^{+}$and $\mathrm{N}_{2} \mathrm{D}^{+}(3-2)$ molecular lines. From this plot we see that the velocity dispersions of these two high-density tracers agree well.

\section{Discussion and conclusion}

As stated in Sect.1, we aimed to test whether the previous $\mathrm{N}_{2} \mathrm{H}^{+}(1-0)$ observations of the Spokes cluster were in fact probing the larger extended emission and not the densest gas in the star-forming cores. If this scenario is correct, we would expect the linewidths of higher-J transition emission, tracing higherdensity gas, to be narrower. The results presented in the previous section indicate that the denser gas does indeed present narrower linewidths. For $\mathrm{N}_{2} \mathrm{H}^{+}(1-0)$ lines, as previously discussed in Peretto et al. (2006), the nonthermal velocity dispersions are supersonic and in most of them the $\sigma_{\mathrm{NT}}>2 c_{\mathrm{s}}(10 \mathrm{~K})$. However, in the higher-density gas, traced by $\mathrm{N}_{2} \mathrm{H}^{+}(3-2)$, the nonthermal velocity dispersion is lower than that of $\mathrm{N}_{2} \mathrm{H}^{+}(1-0)$. In fact, 

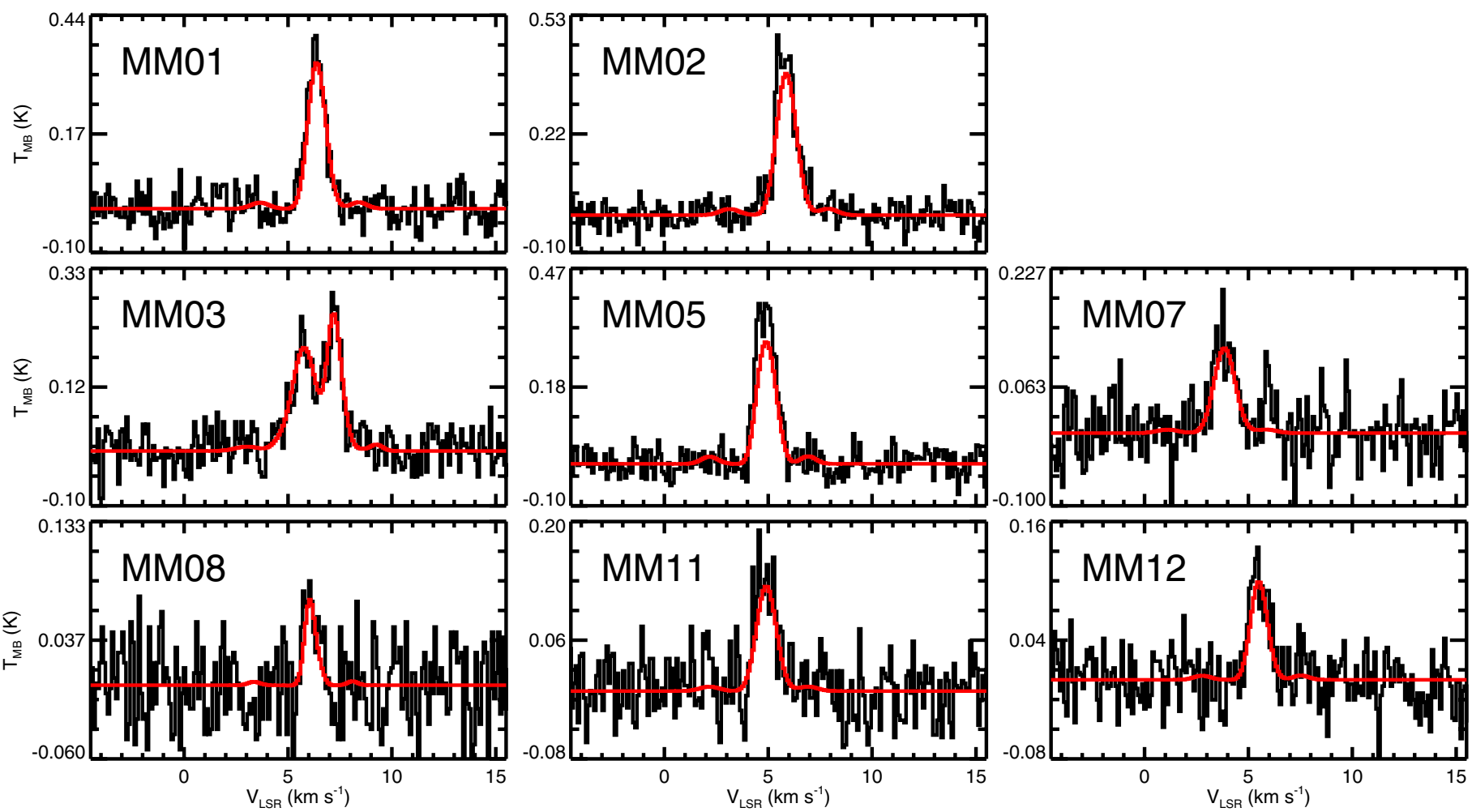

Fig. 3. Observed $\mathrm{N}_{2} \mathrm{D}^{+}(3-2)$ spectra toward the sources. The red curve is the best-fit model to the observations, whose fit parameters are shown in Table 3.

Table 3. Best-fit line parameters for sources in NGC 2264-D.

\begin{tabular}{|c|c|c|c|c|c|c|c|c|c|}
\hline \multirow[b]{2}{*}{ Source $^{a}$} & \multicolumn{5}{|c|}{$\mathrm{N}_{2} \mathrm{H}^{+}(3-2)$} & \multicolumn{4}{|c|}{$\mathrm{N}_{2} \mathrm{D}^{+}(3-2)$} \\
\hline & $\begin{array}{l}T_{\mathrm{ex}} \\
(\mathrm{K})\end{array}$ & $\begin{array}{c}V_{\mathrm{LSR}} \\
\left(\mathrm{km} \mathrm{s}^{-1}\right)\end{array}$ & $\begin{array}{c}\sigma_{v} \\
\left(\mathrm{~km} \mathrm{~s}^{-1}\right)\end{array}$ & $\tau_{\text {main }}$ & $\begin{array}{c}W \\
\left(\mathrm{~K} \mathrm{~km} \mathrm{~s}^{-1}\right)\end{array}$ & $\begin{array}{c}V_{\mathrm{LSR}} \\
\left(\mathrm{km} \mathrm{s}^{-1}\right)\end{array}$ & $\begin{array}{c}\sigma_{v} \\
\left(\mathrm{~km} \mathrm{~s}^{-1}\right)\end{array}$ & $\tau_{\text {main }}$ & $\begin{array}{c}W \\
\left(\mathrm{~K} \mathrm{~km} \mathrm{~s}^{-1}\right)\end{array}$ \\
\hline MM01 & $10.90 \pm 0.03$ & $6.405 \pm 0.004$ & $0.495 \pm 0.003$ & $8.0 \pm 0.2$ & $16.07 \pm 0.08$ & $6.26 \pm 0.02$ & $0.38 \pm 0.02$ & $<0.07$ & $0.43 \pm 0.03$ \\
\hline MM02 & $7.37 \pm 0.05$ & $5.713 \pm 0.009$ & $0.339 \pm 0.007$ & $10.5 \pm 0.6$ & $5.9 \pm 0.1$ & $5.75 \pm 0.02$ & $0.43 \pm 0.02$ & $<0.19$ & $0.57 \pm 0.02$ \\
\hline MM03a & $5.6 \pm 0.2$ & $7.15 \pm 0.02$ & $0.22 \pm 0.03$ & $4 \pm 1$ & $5.89 \pm 0.09$ & $7.11 \pm 0.03$ & $0.28 \pm 0.03$ & $<0.27$ & $0.52 \pm 0.03$ \\
\hline MM03b & 6.7 & $6.25 \pm 0.05$ & $1.33 \pm 0.04$ & $<0.90$ & $b$ & $5.65 \pm 0.05$ & $0.54 \pm 0.06$ & $<0.11$ & \\
\hline MM04 & $6.34 \pm 0.07$ & $4.75 \pm 0.02$ & $0.61 \pm 0.02$ & $4.2 \pm 0.5$ & $4.8 \pm 0.1$ & & & & \\
\hline MM05 & $4.25 \pm 0.03$ & $4.72 \pm 0.02$ & $0.33 \pm 0.02$ & $8 \pm 1$ & $1.04 \pm 0.04$ & $4.79 \pm 0.02$ & $0.36 \pm 0.02$ & $<1.04$ & $0.50 \pm 0.03$ \\
\hline MM06 & $6.58 \pm 0.03$ & $5.694 \pm 0.007$ & $0.349 \pm 0.007$ & $6.9 \pm 0.4$ & $3.96 \pm 0.06$ & & & $\ldots$ & $\ldots$ \\
\hline MM07 & $8.53 \pm 0.03$ & $3.366 \pm 0.005$ & $0.452 \pm 0.005$ & $4.7 \pm 0.2$ & $7.53 \pm 0.06$ & $3.72 \pm 0.07$ & $0.47 \pm 0.07$ & $<0.04$ & $0.19 \pm 0.03$ \\
\hline MM08 & $6.17 \pm 0.05$ & $6.75 \pm 0.01$ & $0.65 \pm 0.02$ & $3.9 \pm 0.4$ & $4.59 \pm 0.06$ & $5.97 \pm 0.06$ & $0.23 \pm 0.07$ & $<0.06$ & $0.07 \pm 0.03$ \\
\hline MM09 & $7.74 \pm 0.03$ & $6.103 \pm 0.006$ & $0.436 \pm 0.005$ & $6.4 \pm 0.2$ & $6.76 \pm 0.06$ & $\ldots$ & $\ldots$ & $\ldots$ & $\ldots$ \\
\hline MM10 & $6.32 \pm 0.04$ & $6.66 \pm 0.01$ & $0.51 \pm 0.01$ & $5.0 \pm 0.4$ & $4.38 \pm 0.06$ & & & $\ldots$ & $\ldots$ \\
\hline MM11 & 6.7 & $4.72 \pm 0.05$ & $0.50 \pm 0.05$ & $<0.11$ & $0.27 \pm 0.03$ & $4.78 \pm 0.05$ & $0.43 \pm 0.06$ & $<0.07$ & $0.19 \pm 0.03$ \\
\hline MM12 & 6.7 & $5.31 \pm 0.02$ & $0.39 \pm 0.02$ & $<0.52$ & $0.89 \pm 0.04$ & $5.38 \pm 0.05$ & $0.36 \pm 0.05$ & $<0.06$ & $0.13 \pm 0.02$ \\
\hline MM13a & 6.7 & $4.20 \pm 0.02$ & $0.42 \pm 0.02$ & $<1.20$ & $3.14 \pm 0.07$ & $\ldots$ & $\ldots$ & $\ldots$ & $\ldots$ \\
\hline MM13b & 6.7 & $5.84 \pm 0.03$ & $0.49 \pm 0.03$ & $<0.61$ &.$^{b}$ & . & $\ldots$ & $\ldots$ & $\ldots$ \\
\hline MM15 & $4.18 \pm 0.05$ & $5.85 \pm 0.02$ & $0.38 \pm 0.03$ & $5 \pm 1$ & $0.89 \pm 0.05$ & $\ldots$ & $\ldots$ & $\ldots$ & $\ldots$ \\
\hline
\end{tabular}

Notes. ${ }^{(a)}$ If the spectrum shows two components, they were both fitted, listed in different rows, and labeled as components a and b. ${ }^{(b)}$ We were unable to derive $\mathrm{W}$ for each of the two components - the value listed for component a corresponds to the integrated W for both components.

the $\mathrm{N}_{2} \mathrm{H}^{+}(3-2)$ velocity dispersions are $\approx 70 \%$ of the value derived from $\mathrm{N}_{2} \mathrm{H}^{+}(1-0)$, and more than half of the total sample shows $\sigma_{\mathrm{NT}} \leq 2 c_{\mathrm{s}}(10 \mathrm{~K})$. There are, however, three outlier data points where the velocity dispersion derived from $\mathrm{N}_{2} \mathrm{H}^{+}(3-2)$ is higher than those from $\mathrm{N}_{2} \mathrm{H}^{+}(1-0)$. Close inspection reveals that a) one of these outliers corresponds to a poorly constrained second component (MM03b); b) another outlier presents asymmetries in the line profile that clearly suggests the presence of a second unresolved component along the line-of-sight (MM08), and finally; c) the remaining outlier data point is obtained from the spectrum with the lowest signal-to-noise ratio with a large associated uncertainty (MM11). Finally, the velocity dispersions obtained with $\mathrm{N}_{2} \mathrm{D}^{+}(3-2)$ either agree with those obtained with $\mathrm{N}_{2} \mathrm{H}^{+}(3-2)$ or are lower. It is important to note there are two cores in our sample that show velocity dispersions consistent (within the 1- $\sigma$ uncertainties) with a sonic nonthermal component: MM03a in $\mathrm{N}_{2} \mathrm{H}^{+}(3-2)$ and MM08 in $\mathrm{N}_{2} \mathrm{D}^{+}(3-2)$.

These results thus support the thermal-fragmentation scenario presented in Teixeira et al. (2006). Although the nonthermal velocity dispersions here reported are still broad, they are much narrower than those found previously using $\mathrm{N}_{2} \mathrm{H}^{+}(1-0)$ single-dish observations (Peretto et al. 2006). It also is important 


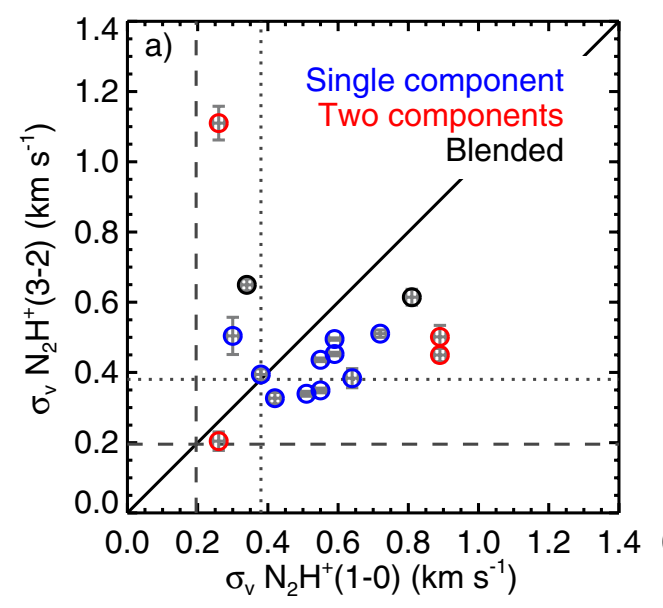

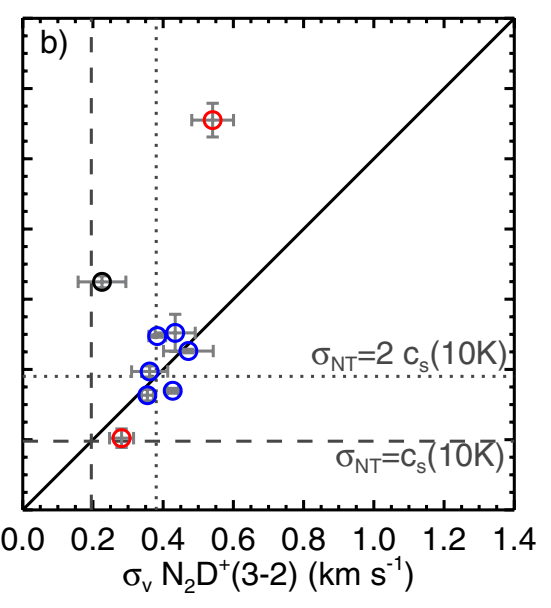

Fig. 4. Comparison of the velocity dispersion of different molecular lines. Cores listed as having two components (see Table 3 ) are shown as red symbols, cores that appear to have blended components are shown in black, and the rest are shown as blue symbols. Dashed and dotted lines show the expected velocity dispersion for the cases where the nonthermal velocity dispersion, $\sigma_{\mathrm{NT}}$, equals $c_{\mathrm{s}}(10 \mathrm{~K})$ or $2 c_{\mathrm{s}}(10 \mathrm{~K})$, where $c_{\mathrm{S}}(10 \mathrm{~K})$ is the sound speed of the average molecule at $10 \mathrm{~K}$ (for a mean molecular weight of $\mu_{m}=2.33$ ).
Table 4. Column densities.

\begin{tabular}{lccc}
\hline \hline Source & $\begin{array}{c}N\left(\mathrm{~N}_{2} \mathrm{H}^{+}\right) \\
\left(10^{12} \mathrm{~cm}^{-2}\right)\end{array}$ & $\begin{array}{c}N\left(\mathrm{~N}_{2} \mathrm{D}^{+}\right) \\
\left(10^{11} \mathrm{~cm}^{-2}\right)\end{array}$ & $N\left(\mathrm{~N}_{2} \mathrm{D}^{+}\right) / N\left(\mathrm{~N}_{2} \mathrm{H}^{+}\right)$ \\
\hline MM01 & $58 \pm 1$ & $4.4 \pm 0.3$ & $0.0076 \pm 0.0006$ \\
MM02 & $56 \pm 4$ & $11.2 \pm 0.5$ & $0.020 \pm 0.002$ \\
MM03 & $19.5 \pm 0.3$ & $12.7 \pm 0.7$ & $0.065 \pm 0.004$ \\
MM04 & $45 \pm 6$ & $\ldots$ & $\ldots$ \\
MM05 & $86 \pm 14$ & $65 \pm 3$ & $0.08 \pm 0.01$ \\
MM06 & $41 \pm 3$ & $\ldots$ & $\ldots$ \\
MM07 & $31 \pm 1$ & $2.7 \pm 0.4$ & $0.009 \pm 0.002$ \\
MM08 & $46 \pm 5$ & $2.0 \pm 0.9$ & $0.004 \pm 0.002$ \\
MM09 & $42 \pm 2$ & $\ldots$ & $\ldots$ \\
MM10 & $45 \pm 3$ & $\ldots$ & $\ldots$ \\
MM11 & $0.9 \pm 0.1$ & $4.6 \pm 0.7$ & $0.5 \pm 0.1$ \\
MM12 & $3.0 \pm 0.1$ & $3.2 \pm 0.5$ & $0.11 \pm 0.02$ \\
MM13 & $10.4 \pm 0.2$ & $\ldots$ & $\ldots$ \\
MM15 & $60 \pm 15$ & $\ldots$ & $\ldots$ \\
\hline
\end{tabular}

Notes. ${ }^{(\dagger)}$ The spectra of sources NGC 2264D-MM03 and -MM13 show two components, but since the optically thin model had to be used for at least one of the components, we used the optically thin column density estimate.

to note that these single-dish observations have a coarse angular resolution $(\approx 17840 \mathrm{AU}=0.086 \mathrm{pc}$ at $800 \mathrm{pc})$, and the substructure or several components along the line-of-sight could increase the observed linewidth. It is clear that more high-angular resolution interferometric observations using high-density tracers are needed to truly assess the kinematics and substructure within NGC 2264-D.

Deuteration can be used as a proxy for the chemical evolution of a core (Crapsi et al. 2005; Emprechtinger et al. 2009; Fontani et al. 2011). For prestellar cores, the deuteration fraction increases as these cores evolve, reaching a maximum at the onset of star formation; the deuteration fraction of the core then begins to decrease as the protostar evolves. For this reason, a high-deuteration fraction in a protostellar core is a particular indicator of youth of the protostar, and a high-deuteration fraction of a prestellar core is an indicator that the core may soon collapse to form a protostar. Table 4 shows that there are four cores (MM03, MM05, MM11, and MM12) with a deuteration fraction at least a factor of 3 higher than the rest of the sample, where the largest difference is a factor of 125. Three of these cores are spatially separated from the main cluster (see Fig. 1) and are embedded in a less dense region. Furthermore, Table 1 shows that no protostar is identified in MM11, and both MM05 and MM12 have faint $24 \mu \mathrm{m}$ sources that have no counterparts at other infrared wavelengths, meaning that these two cores could potentially be harboring either very young or very low mass protostars (additional investigation is necessary to confirm the protostellar nature of MM05 and MM12). Our results thus suggest that these three cores are the least evolved in the Spokes cluster, i.e., closest to the very earliest star formation evolutionary phase. Friesen et al. (2010) found similar results in their analysis of the cores in the Ophiuchus B2 region, where the deuteration fraction decreases with proximity to protostellar cores. The spectrum of core MM03, which is located within the dense region of the Spokes cluster, shows two components, as mentioned. Additional investigation, requiring higher angular and spectral resolution data, is needed to understand the deuteration fraction in this particular core. Moreover, we found no correlation between the level of deuteration and velocity dispersion. Although these few data points make this result uncertain, they agree with what was found in Friesen et al. (2013) in a larger study of nearby low-mass cores.

\section{Summary}

We summarize our main results and conclusions as follows:

1. We observed in the Spokes cluster 14 cores in $\mathrm{N}_{2} \mathrm{H}^{+}(3-2)$ and 8 cores in $\mathrm{N}_{2} \mathrm{D}^{+}(3-2)$ with APEX; the measured linewidths are overall narrower than those of previous $\mathrm{N}_{2} \mathrm{H}^{+}(1-0)$ observations, $\sigma_{v}\left[\mathrm{~N}_{2} \mathrm{H}^{+}(3-2)\right] \sim$ $0.7 \sigma_{v}\left[\mathrm{~N}_{2} \mathrm{H}^{+}(1-0)\right]$.

2. The three cores (MM03b, MM08, and MM11) that do not show narrower $\mathrm{N}_{2} \mathrm{H}^{+}(3-2)$ linewidths than the $\mathrm{N}_{2} \mathrm{H}^{+}(1-0)$ linewidths are cores with more than one component along the line-of-sight or low signal-to-noise spectra.

3. The denser gas, probed by higher J-transitions of $\mathrm{N}_{2} \mathrm{H}^{+}$and $\mathrm{N}_{2} \mathrm{D}^{+}$, presents lower levels of turbulence (nonthermal velocity dispersion) and in two cases approaches the sound speed. These results support the scenario of thermal fragmentation in the expectation that higher-angular resolution observations and/or higher-density tracers would find more quiescent gas.

4. Finally, we find that the three cores of our sample, spatially separated from the main Spokes cluster, are likely the youngest, based on their higher deuteration values.

Acknowledgements. We thank Gary Fuller, Charlie Lada, Paola Caselli, and the anonymous referee for comments that improved the paper. We also thank the staff at the APEX telescope for performing the observations presented in this paper in service-mode. J.E.P. has received funding from the European Communitys Seventh Framework Programme (/FP7/2007-2013/) under grant agreement No. 229517. This publication is supported by the Austrian Science Fund (FWF). 


\section{Appendix A: Column density determination}

The column density is calculated for the optically thick and thin cases using different expressions, but in both cases assuming a beam filling factor of 1 . In the optically thick case,

$$
\begin{aligned}
N_{\text {Tot }}= & 2.01 \times 10^{13}\left(\frac{\sigma_{v}}{\mathrm{~km} \mathrm{~s}^{-1}}\right)\left(\frac{\mu}{\text { Debye }}\right)^{-2} \frac{1}{(J+1)} \\
& \times \frac{\tau}{\left(1-\mathrm{e}^{-T_{0} / T_{\text {ex }}}\right)} \frac{Q_{\text {rot }}(T)}{\mathrm{e}^{-J(J+1) h B / k T_{\text {ex }}}}
\end{aligned}
$$

and in the optically thin case,

$$
\begin{aligned}
N_{\text {Tot }}= & 8.01 \times 10^{12}\left(\frac{W}{\mathrm{Kkm} \mathrm{s}^{-1}}\right)\left(\frac{\mu}{\text { Debye }}\right)^{-2} \frac{1}{(J+1)} \\
& \times\left[\frac{1 \mathrm{~K}}{J_{v}\left(T_{\mathrm{ex}}\right)-J_{v}\left(T_{\mathrm{bg}}\right)}\right] \frac{1}{\left(1-\mathrm{e}^{-T_{0} / T_{\mathrm{ex}}}\right)} \frac{Q_{\mathrm{rot}}(T)}{\mathrm{e}^{-J(J+1) h B / k T_{\mathrm{ex}}}},
\end{aligned}
$$

which are the same as Eqs. (A.1) and (A.4) in Caselli et al. (2002) after rearranging terms and using that

$$
\begin{aligned}
A_{J+1 \rightarrow J}= & \frac{64 \pi^{4}}{3 h c} v^{3}\left|\mu_{J+1 \rightarrow J}\right|^{2}=1.164 \\
& \times 10^{-11}\left(\frac{v}{\mathrm{GHz}}\right)^{3}\left(\frac{\mu}{\text { Debye }}\right)^{2}\left(\frac{J+1}{2 J+3}\right) \mathrm{s}^{-1} \\
\left|\mu_{J+1 \rightarrow J}\right|^{2}= & \mu^{2} \frac{J+1}{2 J+3} \\
g_{J}= & 2 J+1 \\
E_{J}= & J(J+1) h B \\
Q_{\mathrm{rot}}(T)= & \sum_{J=0}^{\infty}(2 J+1) \mathrm{e}^{-J(J+1) h B / k T},
\end{aligned}
$$

where $T_{0} \equiv h v / k$ and $J_{v}(T)=T_{0} /\left[\exp \left(T_{0} / T\right)-1\right]$. The partition function is calculated using the first 100 levels.

\section{References}

Arce, H. G., Borkin, M. A., Goodman, A. A., Pineda, J. E., \& Halle, M. W. 2010, ApJ, 715, 1170

Arce, H. G., Borkin, M. A., Goodman, A. A., Pineda, J. E., \& Beaumont, C. N. 2011, ApJ, 742, 105

Bate, M. R. 2009, MNRAS, 392, 590

Baxter, E. J., Covey, K. R., Muench, A. A., et al. 2009, AJ, 138, 963

Caselli, P. 2002, Planet. Space Sci., 50, 1133

Caselli, P., Walmsley, C. M., Zucconi, A., et al. 2002, ApJ, 565, 344

Crapsi, A., Caselli, P., Walmsley, C. M., et al. 2005, ApJ, 619, 379

Dahm, S. E., \& Simon, T. 2005, AJ, 129, 829

Emprechtinger, M., Caselli, P., Volgenau, N. H., Stutzki, J., \& Wiedner, M. C. 2009, A\&A, 493, 89

Fontani, F., Palau, A., Caselli, P., et al. 2011, A\&A, 529, L7

Fontani, F., Palau, A., Busquet, G., et al. 2012, MNRAS, 423, 1691

Foster, J. B., Rosolowsky, E. W., Kauffmann, J., et al. 2009, ApJ, 696, 298

Friesen, R. K., Di Francesco, J., Myers, P. C., et al. 2010, ApJ, 718, 666

Friesen, R. K., Kirk, H. M., \& Shirley, Y. L. 2013, ApJ, 765, 59

Fuller, G. A., \& Myers, P. C. 1992, ApJ, 384, 523

Goodman, A. A., Barranco, J. A., Wilner, D. J., \& Heyer, M. H. 1998, ApJ, 504, 223

Ho, P. T. P., Moran, J. M., \& Lo, K. Y. 2004, ApJ, 616, L1

Li, Z.-Y., \& Nakamura, F. 2006, ApJ, 640, L187

Markwardt, C. B. 2009, in Astronomical Data Analysis Software and Systems XVIII, eds. D. A. Bohlender, D. Durand, \& P. Dowler, ASP Conf. Ser., 411, 251

McKee, C. F., \& Ostriker, E. C. 2007, ARA\&A, 45, 565

Myers, P. C. 1983, ApJ, 270, 105

Naylor, T., \& Mayne, N. J. 2010, Highlights of Astronomy, 15, 763

Palau, A., Estalella, R., Ho, P. T. P., Beuther, H., \& Beltrán, M. T. 2007, A\&A, 474,911

Peretto, N., André, P., \& Belloche, A. 2006, A\&A, 445, 979

Pineda, J. E., Goodman, A. A., Arce, H. G., et al. 2010, ApJ, 712, L116

Pineda, J. E., Goodman, A. A., Arce, H. G., et al. 2011, ApJ, 739, L2

Teixeira, P. S., Lada, C. J., Young, E. T., et al. 2006, ApJ, 636, L45

Teixeira, P. S., Zapata, L. A., \& Lada, C. J. 2007, ApJ, 667, L179

Vassilev, V., Meledin, D., Lapkin, I., et al. 2008, A\&A, 490, 1157

Ward-Thompson, D., Zylka, R., Mezger, P. G., \& Sievers, A. W. 2000, A\&A, 355,1122

Williams, J. P., \& Garland, C. A. 2002, ApJ, 568, 259

Wolf-Chase, G., Moriarty-Schieven, G., Fich, M., \& Barsony, M. 2003, MNRAS, 344, 809 